\title{
Schaffner's Model of Theory Reduction: Critique and Reconstruction*
}

\author{
Rasmus Grønfeldt Winther†
}

Schaffner's model of theory reduction has played an important role in philosophy of science and philosophy of biology. Here, the model is found to be problematic because of an internal tension. Indeed, standard antireductionist external criticisms concerning reduction functions and laws in biology do not provide a full picture of the limits of Schaffner's model. However, despite the internal tension, his model usefully highlights the importance of regulative ideals associated with the search for derivational, and embedding, deductive relations among mathematical structures in theoretical biology. A reconstructed Schaffnerian model could therefore shed light on mathematical theory development in the biological sciences and on the epistemology of mathematical practices more generally.

1. Introduction. The general model of theory reduction presented by Kenneth Schaffner (1967, 1969, 1976, 1993a, 1993b) has played an important role in the literature on reduction. It provided a clear logical empiricist account of theory reduction. It also served as a counterpoint for critics to develop alternative views of reduction and antireduction. In this paper I wish to complement previous literature on Schaffner's model in two ways. First, I articulate a previously unexplored critique that arises from noticing a tension internal to the model. The model fails on its own terms. Indeed, previous external criticisms regarding the model's logical empiricist assumptions about laws and reduction functions provide an inadequate and incomplete picture of the weaknesses of the model.

*Received November 2006; revised March 2009.

$\dagger$ To contact the author, please write to: Philosophy Department, University of California, Santa Cruz, 1156 High St., Santa Cruz, CA 95064; e-mail: rgw@ucsc.edu.

¥I gratefully acknowledge the suggestions on earlier drafts provided by Elena ÁlvarezBuylla, Michael Dickson, Paul Griffiths, David Hull, Alan Love, Sergio Martínez, Fabrizzio Guerrero McManus, Yuriditzi Pascacio, Alexander Rosenberg, Kenneth Schaffner, Kenneth Waters, and two anonymous reviewers. This research was funded in part by a faculty research grant from the Academic Senate Committee on Research, University of California, Santa Cruz.

Philosophy of Science, 76 (April 2009) pp. 119-142. 0031-8248/2009/7602-0002\$10.00 Copyright 2009 by the Philosophy of Science Association. All rights reserved. 
Second, I advocate reconstructing a Schaffner-like model. Some types of mathematical laws and reduction functions are pertinent to theoretical biology. Moreover, theoretical biology (e.g., parts of evolutionary theory and systems theory) obeys important regulative ideals concerning the search for derivational, and embedding, deductive relations among abstract mathematical structures. In this context, distinct levels of biological organization are often associated with different types of mathematical structures (e.g., models of gene regulatory networks and models of morphological development). Since Schaffner's model captures laws, reduction functions, and the regulative ideal better than other available accounts of theory reduction, reconstructing something like it is worthwhile. Such a model would also shed light on the epistemology of mathematical practices.

This paper is structured as follows. For the remainder of this section, I document the importance of Schaffner's model. I also present a skeleton version of my critique. In Section 2, I elucidate the model. Section 3 explores the internal critique. Section 4 evaluates whether there is any escape for Schaffner's model. Section 5 assesses the relevance of a reconstructed mathematical account of theory reduction for contemporary theoretical biology.

1.1. The Continuing Relevance of Schaffner's Model. Schaffner's account continues to be discussed. Batterman (2005) contends that Schaffner's analysis is a "sophisticated Nagelian type schema." In the context of proposing a new unified gene concept, Beurton claims that Schaffner's views on general reduction are shared by "most molecular biologists" (2000, 308). Third, Sarkar (1998) and Brigandt and Love (2008) examine Schaffner's model at great length.

Why should this be? After all, the standard view seems to be that Schaffner's model is inadequate since biology lacks theoretical laws from which to deductively derive anything. Current proposals of reduction focus on mechanistic, causal or ontological reduction (e.g., Sarkar 1992, 1998; Rosenberg 1997, 2006; Darden and Tabery 2005; Weber 2005; Wimsatt 2007; Brigandt and Love 2008). In general, theory reduction seems to have been abandoned in the philosophy of biology as a viable option.

However, other efforts in the philosophy of science provide two reasons for holding that this model is not a dead letter. First, recent work defends the existence of mathematical laws in evolution and ecology (e.g., Cooper 1996; Brandon 1997; Sober 1997; Waters 1998; Lange 2000). These analyses taken in conjunction with some recent scientific work in evolutionary theory and systems biology (with roots in systems theory) suggests that we should accept the existence of a theoretical, mathematical biology (e.g., 
Okasha 2007; Winther 2008). External critiques of Schaffner's model that do so by rejecting laws tout court may therefore miss their mark.

Second, Schaffner's model captures important scientific norms regarding derivational and embedding relations, which are also connected to unification (e.g., Friedman 1974, 1983; Kitcher 1981, 1989, 1999; van Fraassen 1989; Halonen and Hintikka 2005). These ideals are operative in theoretical biology. Given that Schaffner's model of reduction respects these ideals as well as the pertinence of laws for theoretical biology, it deserves our continued attention.

1.2. An Internal Critique of Schaffner's Model. Very briefly, here is the internal critique. Two desiderata are essential to his model: (1) effecting a heterogeneous reduction between two conceptually distant theories and (2) satisfying the sets of closeness constraints pertinent to both deductive inference and theory correction. I show that both sets of closeness constraints cannot be simultaneously satisfied given significant conceptual distance. The constraints are in tension.

2. The Basics of Schaffner's Model. The basic logical empiricist account of reduction views scientific explanation as a deductive relation between theories (e.g., Hempel and Oppenheim 1948; Nagel 1949, 1961, 1979; Hempel 1966). For a theory $T_{1}$ to reduce a theory $T_{2}$ is for $T_{2}$ to be deducible from $T_{1}$ :

$$
T_{1} \vdash T_{2} \text {. }
$$

Following convention, we call $T_{1}$ the 'reducing theory' and $T_{2}$ the 'reduced theory'. Nagel distinguishes between homogeneous and heterogeneous reduction. Homogeneous reduction "generates no special logical puzzles" $(1961,339)$ because the two theories have similar formalizations and also contain directly connectable, even identical, terms. Terms are the objects, predicates, and relations pertinent to the theory. But heterogeneous reduction does elicit philosophical problems since the two theories differ in their formalizations and terms. Moreover, they are conceptually distant.

The basic logical empiricist account holds that a theory consists roughly of theoretical and observational components: (1) axiomatic theoretical laws, and experimental-observational laws, (2) theoretical and observational vocabularies, and (3) bridge principles connecting theoretical and observational components (e.g., Hempel 1966). Nagel makes the existence of (1) laws and (2) vocabularies the first two "formal conditions for re- 
duction" (1961, 345-351). ${ }^{1}$ Nagel's third and last explicit condition for reduction requires the background structure given by the first two conditions. This third condition consists of two "formal requirements that must be satisfied for the reduction of one science to another" (352): derivability and connectability. Bridge principles are the "assumptions" (352353) that ground these two formal requirements. Again, the requirements are trivially satisfied for homogeneous reductions. However, for heterogeneous reductions, satisfying them is challenging; communal effort over historical time is required.

The "condition of derivability" (354) requires that the laws and theoretical assumptions of the reduced science must be deductively derivable from those of the reducing science (Nagel 1961, 354). Indeed, there are many important similarities between Hempel's Deductive-Nomological model of explanation and Nagel's account of theory reduction.

The "condition of connectability" requires that the terms of the reduced theory be connected to the terms of the reducing theory (Nagel 1961, 353-354). Derivability entails connectability, but not vice versa (355). Nagel (1961) is, unfortunately, none too concerned with specifying the nature of these connections. ${ }^{2}$ He simply notes that "assumptions of some kind must be introduced" in order to connect the terms of the two theories (353). Such "assumptions" have been named "bridge principles," "bridge laws," and "correspondence rules" (e.g., Nagel 1961, Hempel 1966, Kitcher 1984, Sarkar 1998). I follow Schaffner and call them 'reduction functions'. This terminology reminds us that the principles and rules justifying the connections are intended to be functions in the technical sense, subject to the formal constraints of mapping elements in the domain (set of all inputs) to elements in the range (set of all outputs). Schaffner's view is even stronger. Each reduction function must be extensionally injective (one-to-one) and surjective (onto), that is, it must be bijective. By focusing on relations across theories, this terminology also disambiguates two senses of 'bridging': (1) linking theoretical and observational terms of the same theory (e.g., Hempel's main use), or (2) connecting terms, of either vocabulary, across theories (e.g., Nagel's and Schaffner's primary use).

1. Nagel observes that informal, pragmatic conditions must also be met for a theory reduction of the form [1] to be scientifically interesting. Since Schaffner agrees with these pragmatic conditions, I will not consider them further.

2. But see Nagel 1979, 103-107, where he develops a sketch of an extensional account in rough outline similar to Schaffner's account. This chapter is rarely, if ever, cited in the subsequent literature. 
In what follows, I employ Schaffner's sense of reduction functions. ${ }^{3}$ I return to this point in Section 5.

Schaffner concurs with Nagel's two formal conditions. He supplements the formal account by (1) introducing corrected reduced theory and (2) clearly characterizing reduction functions.

First, theory correction. In response to Nagel's account of theory reduction, Feyerabend argues that the reducing and reduced theories are incommensurable. Specifically, the intensions of their respective terms, as well as their respective ontological commitments, are not commensurate. It is impossible to directly connect terms in a reduced science to terms in an uncorrected reducing science. Indeed, putative theory reduction is actually theory replacement (Feyerabend 1962, 1965). Schaffner addresses this problem by introducing the notion of a corrected reduced theory. Roughly, Schaffner holds that the original reduced theory, $T_{2}$, and the corrected reduced theory, $T_{2}^{*}$, belong to a set, $K$, of versions of the same theory:

$$
K=\left\{T_{2}, T_{2}^{*}, T_{2}^{* *}, \ldots\right\} .
$$

Between any pair of theories of the set $K$ there are relations of "approximate equality, close agreement, and strong analogy" (Schaffner 1976, 617; see also 1993a, 429). These are stringent constraints on the similarity between any two versions of the same theory. These closeness constraints define what it is to be part of the set $K{ }^{4}$ Schaffner's response to Feyerabend's incommensurability objection, then, is that the corrected reduced theory $\left(T_{2}^{*}\right)$ serves as the commensurability link between the original reduced $\left(T_{2}\right)$ and reducing $\left(T_{1}\right)$ theory. ${ }^{5}$

What about the correction of the reducing theory? For two reasons, I will not address this here. First, Schaffner rarely mentions it (e.g., Schaffner 1969, 332; 1993a; Hull 1976, 662; and Kitcher 1984, 338, briefly discuss the necessity of reducing theory correction). Second, and more importantly, this kind of theory correction does not make any difference to my argument. In fact, considering it would only make the conceptual distance

3. Nagel (1961) argues that it is actually the theoretical component of $T_{1}$ that reduces the "experimental laws" (and "if it has an adequate theory, its theory as well") of $T_{2}$ (352). However, significant portions of Nagel's argument as well as subsequent discussions on theory reduction bracket this point. Indeed, the promises and pitfalls of intertheory reduction can be addressed without loss of philosophical generality even when the distinction between theoretical and observational components of each theory is set aside.

4. Kenneth Waters provided critical feedback on this point.

5. Another strategy for linking terms in the two theories is to use Hartry Field's (1973) notion of "partial denotation." For a recent related effort, see Psillos 1999 (especially Chapter 12). 
larger or maintain it. Moreover, we would now be concerned with simultaneously satisfying the closeness constraints (1) pertinent to deductive inference from $T_{1}^{*}$ to $T_{2}^{*}$ and (2) those relevant to theory correction of both $T_{1}$ to $T_{1}^{*}$ and $T_{2}$ to $T_{2}^{*}$.

Schaffner also supplements Nagel's account by characterizing reduction functions. According to Schaffner, reduction functions connect equivalent terms of the two theories through coextensional referencing $(1976,614$ 615, 620-624; see discussion in 1993a, 466-477). That is, the extension of a particular term of the corrected reduced theory is identical to the extension of the equivalent term in the reducing theory. Synthetic identities between the vocabularies of the two theories are established. Such reduction functions can be schematically represented thus (see Schaffner 1976, 618; 1993a, 429):

$$
\operatorname{Term}_{i, T 2^{*}}=\text { function }_{i}\left(\operatorname{Term}_{i, T 1}\right) \text {, where } 1 \leq i \leq n .
$$

Here, the terms - that is, the natural kinds - can be of entities, predicates, or relations, or combinations thereof. The total number of terms in the reduced theory is $n$. Each reduction function $i$ has two further, technical properties. Each reduction function must be injective in that every element of the domain term $i$ of the reducing theory maps uniquely to one element of the range term $i$ of the reduced theory. That is, the mapping must be one-to-one, not many-to-one (n.b., one-to-many element relations are already prohibited by the very definition of function). Second, each reduction function must be surjective in that no element of the range term $i$ is extensionally unmapped to an element of the domain term $i$, and vice versa. The reduction functions are thus bijective. The terms are extensionally equivalent. This bijective mapping structure guarantees that every term of the reduced theory has a one-to-one correspondence with a single term of the reducing theory. Now, since the reducing theory is more general, it may contain further terms that map bijectively to terms in other reduced theories. The existence of bijective reduction functions is necessary but not sufficient for the justification of the deduction from the reducing to the reduced theory. Moreover, these reduction functions impose closeness constraints.

Thus, Schaffner's full account of theory reduction can be expressed as

$$
\begin{aligned}
& \left\{T_{1} \text {, \{reduction functions }\right\}, \\
& \qquad \text { various types of initial conditions }\}\} \vdash T_{2}^{*} .
\end{aligned}
$$

Note that the deduction is to the corrected reduced theory (as he indicates in his formalizations in 1976, 618; 1993a, 429), and that a large number and variety of bijective reduction functions are explicitly included in the deduction. Initial conditions such as background assumptions re- 
garding 'normal conditions', must also be expressed and held fixed for the deduction to go through. In contrast, in Nagel's model, there is no explicit notion of a corrected reduced theory and the reduction functions are only implicit.

Before detailing my internal critique, I mention three external criticisms of Schaffner's model. These arose in the context of Schaffner applying his account to genetics. First, a number of commentators argue that molecular genetics does not have any strong, integrated theory (Hull 1972; Wimsatt 1976; Kitcher 1984; Sarkar 1992, 1998). Therefore, Schaffner's account premised on deductive intertheoretical relations must fail. Second, Hull questions the very possibility of providing corrected reduced theories that satisfy Schaffner's closeness constraints. Hull argues that "similarity in substantive content ... is not sufficient for individuating scientific theories. The substantive content of science changes too rapidly and sporadically for that" $(1976,656)$. Instead, he endorses a view individuating scientific theories genealogically, rather than according to substantive content.

Third, it is argued that there are few, if any, one-to-one correspondence reduction functions between classical $\left(T_{2}\right)$ and molecular $\left(T_{1}\right)$ genetics (e.g., Hull 1972, 1974, 1976, 1981, 2002; Rosenberg 1978, 1985, 2002; Kitcher 1984; Sarkar 1998; see Ruse 1976 for a response). There are two separate arguments for the absence of such functions:

1. The reduction functions are extensionally one-to-many in that the elements of (at least some of) the single terms of $T_{1}$ map to elements of two or more terms of $T_{2}$ (no 'type-type reduction'), and each element of $T_{1}$ maps uniquely onto a single element of $T_{2}$ ('tokentoken reduction'). Since mappings exist across the terms of $T_{2}$, such relations cannot be reduction functions in the sense of [3] (Brigandt and Love [2008] call this "context-dependence of molecular features").

2. The reduction functions are extensionally many-to-one in that the elements of (at least some of) the single terms of $T_{2}$ map to elements of two or more terms of $T_{1}$ (no 'type-type reduction') and we accept standard token-token reduction. Again, since mappings occur across terms, such relations cannot be reduction functions in the sense of [3] (this is the "multiple realizability argument"; see Fodor 1974, Kim 1992; Sober 1999).

Because of these two criticisms, neither the connectability condition nor the derivability condition can be satisfied (but see Waters 1990, 2000 on connectability). In short, Schaffner insists on the bijectivity of each ideal pair of terms and on type-type reduction. His critics deny bijectivity (and type-type reduction), while accepting token-token reduction. Antireduc- 
tionist critics claim that there are one-to-many and many-to-one extensional mappings across pairs of actual terms in $T_{1}$ and $T_{2}$.

I bracket the first two external criticisms since my critique is internal. I will return to the third worry because even an internal critique must consider it explicitly.

3. The Internal Critique. The internal critique of Schaffner's account arises from noticing a tension regarding closeness constraints. Given a large conceptual distance, if the corrected reduced theory $\left(T_{2}^{*}\right)$ is of the same type as the original reduced theory $\left(T_{2}\right)$ (i.e., they both belong to the set $K$ of [2]), thereby satisfying closeness constraints on theory correction, it will not be possible to articulate simple, bijective reduction functions. Conversely, in so far as suitable reduction functions can be expressed, thereby satisfying closeness constraints on deductive inference, the corrected reduced theory will not be of the same type as the original reduced theory. In short, both sets of closeness constraints cannot be met simultaneously when a large conceptual distance is to be covered. Satisfy one constraint, and you will not be able to satisfy the other. The constraints are in tension, even by the model's own standards. I justify my critique by further exploration of the two sets of closeness constraints and the idea of conceptual distance.

Consider theory correction. Schaffner does not suggest methods, formal or otherwise, for such transformations. Sarkar observes that a "controversial element" in Schaffner's model is that "there seemed to be no context-independent characterization of the relation between $T_{2}$ and $T_{2}^{*} \ldots$., let alone a formal one. In such a circumstance, no model of reduction that relies only on formal criteria for delineating theories and relations between them can be constructed" (Sarkar 1998, 27; formalism changed for consistency). Indeed, for Schaffner the relation between $T_{2}$ and $T_{2}^{*}$ is "informal" $(1976,617)$. It remains unclear whether any characterization of theory correction consistent with logical empiricist assumptions could be given. Despite this external criticism, Schaffner insists that theory correction can be done and that there are closeness constraints. He states: "traditional genetics $\left(T_{2}\right)$ is not radically reanalyzed; it is rather corrected and enriched" (1993a, 445). Original and corrected theories must be strongly similar, in both formal and substantive respects. ${ }^{6}$

6. It is worth noting that Kitcher does provide an explicit account of how to specify the set $K$ of [2]: "Classical genetics persists as a single theory with different versions at different times in the sense that different practices are linked by a chain of practices along which there are relatively small modifications in language, in accepted questions, and in the patterns for answering questions" $(1984,353)$. Although Kitcher appeals to continuity of practice rather than to theory correction, he seems to maintain that 
Now consider reduction functions of the form of [3]. In discussing the reduction of classical to molecular genetics, Schaffner provides the example of linking the classical genetic term "dominance" to coextensive combinations of molecular genetic (1) predicates: "DNA sequence" and "amino acid sequence" and (2) relations: "chemically causes" (see 1976, 623; 1993a, 442 for details). ${ }^{7}$ This bijective reduction function, with a molecular genetic term that combines molecular predicates and relations in a particular way, would indeed satisfy the closeness constraints on deductive inference. However, it is questionable whether this is a legitimate reduction function. Indeed, Schaffner simply assumes that the molecular basis of dominance will only involve the production of amino acid sequences from DNA, and will furthermore be robust to other causes and background conditions (for criticisms of these assumptions, see Sarkar 1998, 168-173; Bagheri 2005). Despite this external criticism, Schaffner insists on the availability of reduction functions to ground deductions of all the terms of $T_{2}^{*}$. Such functions impose one-to-one correspondence extensional mappings, and thereby force $T_{2}^{*}$ and $T_{1}$ to be close.

Now, the tension between the two closeness constraints arises because the conceptual distance between $T_{1}$ and $T_{2}$ is large. After all, this fact is what makes - or at least, would make - a reduction between them interesting and useful. As Sarkar contends in a more general context: "Unless there is some such difference between where the explanans and the explanada come from, there is little sense in calling an explanation a reduction" (2001, 238). Moreover, Nagel observes that heterogeneous reductions, and not homogeneous reductions, are problematic: "Difficulties are frequently experienced in comprehending the import of a reduction as a consequence of which a set of distinctive traits of some subject matter is assimilated to what is patently a set of quite dissimilar traits. In such cases, the distinctive traits that are the subject matter of the secondary (reduced) science fall into the province of a theory that may have been initially designed for handling qualitatively different materials and that does not even include some of the characteristic descriptive terms of the

different versions of the same theory must be continuous and similar to one another. Furthermore, there is useful literature on constraints on analogical inference (e.g., Gentner 1983; Holyoak and Thagard 1989; Markman 1997). Analogical inference is one possible epistemic manner of correcting theories. Holyoak and Thagard (1989) distinguish among three types of constraints on 'analogical mapping' between a base and a target (e.g., between $T_{2}$ and $T_{2}^{*}$ ): structural consistency, semantic similarity, and pragmatic centrality. Both Kitcher's account and work on analogical inference in cognitive science could provide a more detailed articulation of theory correction and closeness constraints on theory correction.

7. Hull (1976) complains that this is basically the only reduction function Schaffner provides. 
secondary science in its own set of basic theoretical distinctions" (Nagel 1961, 339-340). The two theories have "quite dissimilar traits," handle "qualitatively different materials," "include ... [different] descriptive terms," and have their own "theoretical distinctions." They are conceptually distant. Given the proximity of Schaffner's analysis to Nagel's model, and the general force of these arguments, Schaffner would have to agree that heterogeneous reductions imply a large conceptual distance.

Furthermore, the two theories will be strikingly dissimilar in most actual, historical cases. They have distinct formalizations, key concepts, ontological commitments, historical origins, and developmental routes. Any theory reduction of one to the other would be a nonautomatic, communal accomplishment. This is an empirical argument for the significant conceptual distance between $T_{1}$ and $T_{2}$.

How exactly is my critique internal? Hull, Kitcher, and Rosenberg argue that much of the original meaning (intension) of the term 'dominance' in uncorrected classical genetics is lost when any effective reduction function is articulated. In fact, terms of any $T_{2}$ lose much of their intension as they get transformed and restricted into terms that can ground the deduction of $T_{2}^{*}$ from $T_{1}$. Can I then legitimately grant Schaffner the possibility of stating sufficiently simple reduction functions? If I concede this possibility, then how do I respond to Hull's, Kitcher's, and Rosenberg's worries? If I do not grant it, then how is my critique internal?

In my view, Hull, Kitcher, and Rosenberg are justified in insisting on the necessary complexity of reduction functions if and only if they take the starting points of the reduction to be the uncorrected theories $T_{2}$ and $T_{1}$ (or theories that are extremely close to $T_{1}$ and $T_{2}$ ). But Schaffner need not accept this. Bijective reduction functions can be legitimately attained between $T_{2}^{*}$ and $T_{1}$. Thus, Hull's, Kitcher's, and Rosenberg's criticism are external in that they dismiss Schaffner's two-step model and primarily consider deductive inference of $T_{2}$ from $T_{1}$ (see [1]). In contrast, my critique is internal in that it shows how his account then lacks the resources for dealing with the overall complexity of reducing an uncorrected theory to a reduced uncorrected theory, across a great conceptual distance. That is, contra Hull, Kitcher, and Rosenberg, Schaffnerian reduction functions, which are simple, effective, and bijective, are legitimately possible. However, because of conceptual distance, the simplicity in the reduction function comes at the cost of hiding complexity in (i) other unstated reduction functions not included in [4], (ii) the initial conditions of [4], or (iii) the transformation of $T_{2}$ to $T_{2}^{*}$. Put differently, the external criticisms can be justifiably bracketed for either deductive inference or theory correction, but not for both simultaneously. Indeed, by only considering deductive inference, Hull, Kitcher, and Rosenberg have not identified the full extent of the problem for Schaffner's model. 
To summarize, we have seen that theory correction requires close proximity between $T_{2}$ and $T_{2}^{*}$, whereas deductive inference necessitates a close fit between $T_{2}^{*}$ and $T_{1}$. These respective closeness constraints cannot be satisfied for the same case of heterogeneous reduction.

4. Can the Internal Tension Be Eliminated? There are three possible ways to eliminate the internal tension: (1) deny that there is a large conceptual distance, (2) weaken and qualify the constraints on theory correction, and (3) weaken and qualify the constraints on deductive inference. If we could meet one or more of these, then both sets of closeness constraints could be simultaneously satisfied even with a large conceptual distance. In general, however, none will work.

4.1. Denying a Large Conceptual Distance. Thus far, the discussion has been premised on Schaffner's commitment to a large conceptual distance between theories. There is an ambiguity regarding reduction functions in Schaffner's account that provides a way to deny this assumption. Let us see how.

In Section 3, I argued that reduction functions obtained between the corrected reduced theory and the reducing theory. I call this the CTRF (corrected theory reduction function) interpretation. But another interpretation of Schaffnerian reduction functions is possible. They could be seen to hold between both original theories. I call this the OTRF (original theory reduction function) interpretation.

Let us see how an OTRF interpretation is possible. Schaffner follows "modern logicians such as Quine in construing predicates in an extensional manner. From this perspective, a predicate is simply a class of those things or entities possessing the predicate" $(1976,621)$. How are these set-theoretic predicates linked across two theories? Schaffner answers, "The simplest and least question begging way to do this seems to be to require (i) that the entity terms (e.g., 'gene' or 'DNA') to be associated, be construed as extensionally referring to the same entity, even though that entity is described in different ways and (ii) that predicates and relations (e.g., 'dominant') in the two theories, be interpreted as referring to the same states of affairs, characterized with the help of the entity relations spoken above" (614). Synthetic identity is premised on extensional identity. Intensions are set aside. Extensional equivalence alone provides meaning equivalence.

An OTRF interpretation of Schaffnerian synthetic identities is plausible. Note that Schaffner refers to the "two theories" simpliciter, suggesting that these are uncorrected classical and molecular genetics. Furthermore, in his discussion of reduction functions as "dictionary entries that aid a translator" $(1976,622 ; 1993 a, 441)$, he explicitly holds that the 
functions are between the original reduced and reducing theory (see also, e.g., 1976, 623-624).

Prima facie, it seems that the OTRF interpretation would drastically shrink the conceptual distance since it directly and bijectively maps the extension of the set of terms of $T_{1}$ onto the set of terms of $T_{2}$. Any failure of bijectivity within or across pairs of terms is eliminated. Initially large conceptual distance is diminished through the formalized mappings enforced by Schaffnerian reduction functions. But what about the external criticisms as well as Schaffner's own commitment to a large conceptual distance? Here are three possible ways of characterizing the OTRF interpretation's relevance to conceptual distance. Only the third can legitimately diminish such distance.

(i) The mapping relation between terms in $T_{2}$ and $T_{1}$ is indeed unsuccessful because of concerns with Schaffnerian reduction functions.

(ii) The mapping relation between terms in $T_{2}$ and $T_{1}$ actually collapses to a mapping relation between terms in $T_{2}^{*}$ and $T_{1}$ and we return to my internal critique.

(iii) Same collapsing as in (ii), but the internal critique is avoided because an appropriate middle distance exists for a few cases of pairs of actual theories.

Possibility (i) hardly needs much elaboration. Here the OTRF interpretation is seen as a narrow Nagelian deductive model as represented by [1]. This model is subject to the context-dependence and multiple realizability external critiques that Hull, Kitcher, Rosenberg, Fodor, and Kim forwarded.

But perhaps the OTRF interpretation collapses to the CTRF interpretation. That is, the simple reduction functions might really be between terms in $T_{1}$ and terms in what I call $T_{2(\mathrm{I})}$. The 'I' in $T_{2(\mathrm{I})}$ stands for 'Idealized'. That is, $T_{2(\mathrm{I})}$ is the original $T_{2}$ as acutely idealized by a rational reconstructor of the theory (e.g., Schaffner). The idealization occurs when "the basic principles of the reducing and reduced theories have been codified and . . . the primitive terms of both theories have been determined" (Schaffner 1976, 614). Indeed, terms of $T_{2}$ are reconstructed in light of knowledge of $T_{1}$. This imposition of bijectivity warps the intension and purpose of the terms of $T_{2}$. Moreover, $T_{2(\mathrm{I})}$ is already extremely close to $T_{2}^{*}$ and theory correction happens 'for free'. We return to the CTRF interpretation of Schaffner's two-step model.

Possibilities (ii) and (iii) pertain to this collapse. Either (ii) my original internal critique goes through or (iii) there are some (historically rare) pairs of theories expressing the appropriate amount of conceptual distance such that the reduction between them counts as heterogeneous and the closeness constraints of both forms of inference are also met. Only for 
such middle-distance cases could Schaffner's model be immune to my internal critique.

The internal critique was considered in Section 3 and will not be further discussed here. Now, despite Schaffner's commitment to the significant conceptual distance of interesting heterogeneous reductions, might there not exist middle-distance theories that survive my internal critique as well as the external criticisms (i.e., the context dependency and multiple realizability objections to simple, bijective reduction functions, and worries concerning the possibility of finding appropriate methods for, and criteria of, theory correction)? It is possible. But such theory reductions would be less interesting and would also be historically rare. Moreover, an explicit metric of conceptual space will have to be articulated in order for this issue to be fully addressed. Such a metric is needed to measure the conceptual distance between the reduced theory and the reducing theory, as well as the distance between each of these and $T_{2}^{*}$. The burden of proof seems to lie on a defender of a (reconstructed) Schaffner-like model to make such a metric explicit. In the conclusion, I provide a few tentative suggestions for how to do this in the context of mathematical theory in contemporary theoretical biology. However, my proposal does not pertain to a Schaffnerian set-predicate, extensional logical approach to reduction functions.

For the purposes of the current section, the point is simple: for the most part, large conceptual distance is intrinsic to interesting theory reduction and to most actual scientific theories. Only possibility (iii) can eliminate my internal critique, but this possibility requires further investigation.

4.2. Relaxing Constraints on Theory Correction and Deductive Inference. Two further potential ways of eliminating the internal critique involve relaxing the closeness constraints, in a reasonable manner. By weakening the closeness conditions on theory correction, one would allow more conceptual distance between $T_{2}$ and $T_{2}^{*}$. Alternatively, by debilitating constraints on deduction one would permit more distance between $T_{1}$ and $T_{2}^{*}$. Relaxing either set of constraints would provide more overall conceptual distance and, thereby, alleviate the tension between the closeness constraints.

Weakening the strong analogy, approximate equality, and close agreement constraints may not be possible. First of all, a significantly more explicit formulation of these constraints is required before we could even know how to weaken them (see note 6 above). Moreover, by relaxing them, we would quickly approach the logically dangerous point of blurring the boundaries between theories (e.g., $T_{1}$ and $T_{2}$ ). More generally, there would no longer be meaningful constraints on the similarity among the elements of a given set $K$ in [2]. Finally, any logical empiricist account of 
theory reduction seems to require explicit, stringent, and formal closeness constraints on theory reduction. The denial of these sorts of constraints is, after all, one important motivation for subsequent proposals of nonformal heuristics for theory transformation (see, e.g., Sarkar 1998; Wimsatt 2007). In short, it is unclear whether Schaffner's account can legitimately weaken these constraints.

Alternatively, one could try to relax the formal constraints on deductive inference. However, weakening the constraints on deductive inference does not seem warranted by any logical procedure or criteria. The determination of what counts as a deduction, and judgments of which kinds of reduction functions could possibly be effective in justifying a deduction, are subject to extremely strict criteria, particularly under a logical empiricist account.

Thus, neither of the strategies for relaxing closeness constraints can legitimately increase the conceptual distance between $T_{2}$ and $T_{2}^{*}$ or between $T_{2}^{*}$ and $T_{1}$. Again, the internal tension is not eliminated.

5. Reconstructing Schaffner's Model: Mathematical Reduction in Contemporary Theoretical Biology? Let us take stock. I have shown that there is an internal tension in Schaffner's model of theory reduction. Basically, closeness constraints on theory correction and deductive inference cannot be met simultaneously with covering a large conceptual distance between the reduced and reducing theories. Only in one possible scenario of the OTRF interpretation might my internal critique turn out to be ineffective, but the jury is out on that possibility. Schaffner's model of theory reduction fails, even on its own terms.

My internal critique was explored primarily in the context of the reduction of classical to molecular genetics. But will this critique be relevant for every possible application? Given the general problems investigated in Section 3 and Section 4, it seems that Schaffner's account will fail in other domains as well. Moreover, philosophers of physics have recently provided powerful arguments belying the deductive closeness of phenomenological and statistical thermodynamics (e.g., Callender 1999; Sklar 1999). ${ }^{8}$ Furthermore, this and other paradigmatic cases of reduction in

8. But Shimony (1987) takes issue with this rather pessimistic view. In pointing out that formal derivations in many of the key cases of reduction in physics are "not absolutely general," he notes "that excessive rigidity concerning the concept of reducibility [a rigidity characteristic of Nagel's and Schaffner's models] can mask the truly wonderful relations between levels of description" (418). Orthodox models of theory reduction cannot do full justice to the complexity of intertheoretical relations pertinent to physical theories at different levels. However, a broader understanding of reduction, including the one here sketched, might. I thank Sergio Martínez for discussions of this point. 
theoretical physics involve actual historical theories that have their own formalizations, key concepts, ontological commitments, historical origins, and developmental routes. Although further investigation is required, we suggest that the same internal tension would appear if we were to apply Schaffner's model to cases from the physical sciences.

Despite the deep problems with Schaffner's model, I believe that it deserves our continued attention. A reconstructed Schaffner model could assist us in making sense of the ongoing developments in theoretical biology, particularly in large parts of systems biology and evolutionary theory. There are currently few, if any, available philosophical analyses that can usefully and fairly analyze theory growth, theory reduction, and theory unification in mathematical biology, broadly construed. Rethinking Schaffner's account in light of other philosophical work on mathematical modeling - including the semantic view of theories - gives us a powerful perspective on derivation and embedding in mathematical theorizing and modeling. In what follows, I highlight three aspects of the proposed reconstructive project:

1. The distinction between mathematical and mereological theory reduction.

2. The generalization of deduction to encompass derivational and embedding mathematical practice.

3. The articulation of a metric of conceptual distance.

I first explore (1). After discussing GRNs (gene regulatory networks) in systems biology, I turn to (2) and (3).

Sarkar $(1998,43 f f$.$) distinguishes three substantive criteria for identi-$ fying different forms of reduction: (1) fundamentalism, ${ }^{9}$ (2) abstract hierarchy, and (3) spatial hierarchy. My distinction between mathematical and mereological theory reduction is similar to his differentiation between (2) and (3). Mathematical reduction involves deriving the mathematical models of the reduced theory from those of the reducing theory; to put it in a different, and complementary, fashion, mathematical reduction entails embedding the models of the reduced theory into the models of the reducing theory. Mereological reduction is also theoretical. It occurs when a theoretical representation of higher-level parts of a system is explained in terms of a theoretical representation of lower-level parts (and lower-level relations) of that system.

These two forms of theory reduction are separable. An example of the

9. Sarkar defines "fundamentalism" thus: "the explanation of a feature of a system invokes factors from a different realm (from that of the system, as represented) and the feature to be explained is a result only of the rules operative in that realm" (1998, 43). 
former without the latter is the reduction of classical to relativistic mechanics. Cases of the latter without the former are the "mechanistic reductions" discussed by Craver (2007). Third, when morphogenetic models are reduced to GRNs, both formal and mereological reductions are attained. Schaffner's model simultaneously refers to both forms of reduction. Moreover, a reconstructed Schaffnerian model suggests a connection: the mathematical models of one theory representing a high level of organization are reduced to the mathematical models of another theory representing a mereologically-nested, lower level of organization. For instance, phenotypic mathematical models of development are (partially) reduced to GRNs.

Now that I have briefly characterized mathematical and mereological theory reduction, I turn to systems biology. This will motivate points (2) and (3) of the reconstructive project. The highly mathematical strands of systems biology have roots in earlier systems theory work by Rashevsky (1954, 1961); von Bertalanffy ([1933] 1962, 1968); Goodwin (1963); Rosen (1964, 1985); and Mesarovic (1968). (For reviews, see Kitano 2002; O'Malley and Dupré 2005.) Much of this mathematical work was set aside and ignored starting in the 1970s, perhaps as a consequence of the 'molecular revolution' of biology. But as increasingly sophisticated data sets are made available and as the context dependency and complex nature of biological systems becomes ever more evident, a systems-oriented theoretical approach to biology is reemerging (Winther 2008).

In the context of systems biology, GRNs are garnering significant attention (e.g., Smolen, Baxter, and Byrne 2000; Davidson 2001; De Jong 2002). A variety of modeling methodologies are available. Here I review two important ones: (1) Boolean networks and (2) coupled differential equations.

First, in a Boolean network, a number of elements (genes) are directly connected to one another (e.g., Thomas 1973; Kauffman 1993; Mendoza and Álvarez-Buylla 1998). The state of each element of the system is either 0 ('OFF') or 1 ('ON') and depends on (i) its state one time unit earlier, (ii) the states, one time unit earlier, of the other elements that serve as input to it, and (iii) the Boolean function determining how its state changes as a function of its input elements. Most generally:

$$
\hat{x}_{i}(t+1)=\hat{f}_{i}(\hat{\mathbf{x}}(t)), \quad \text { where } 1 \leq i \leq n,
$$

where $\hat{x}_{\mathrm{i}}$ represents the state of the $i$ th element and $\hat{f}_{\mathrm{i}}$ expresses the Boolean functions mapping inputs to output for each element of the $n$-vector $\hat{\mathbf{x}}$ of states. The $n$ elements of the system determine a state space of $n$ dimensions with $2 \wedge n$ states. The total number of possible Boolean functions is $2 \wedge 2 \wedge k$, where $k$ is the number of inputs. For example, an element 
with 2 inputs $(k=2)$ has 16 possible Boolean functions between its inputs, $x_{1}$ and $x_{2}$. Thus, there are 16 distinct binary Boolean rules, including AND, XNOR, NAND, and material implication. These rules can usefully be expressed in a Hasse diagram (Pemmaraju and Skiena 2003). Boolean networks readily capture the dynamics and topology of genetic connectivity. Here is an example of a single Boolean function (where $n=4$ and $k=2$ ):

$$
\hat{x}_{1}(t+1)=\hat{x}_{2}(t) \operatorname{NAND} \hat{x}_{4}(t) .
$$

By articulating the appropriate sets of Boolean functions, general dynamical regulatory behavior of gene networks can be represented.

Second, coupled differential equations can also be used to model GRNs. Here, differential rate equations express the continuous rate of production of an element as a function of the concentrations of other elements of the system (e.g., Goodwin 1963; Tyson and Othmer 1978; De Jong 2002). Most generally:

$$
d x_{i j} / d t=g_{i j}(\mathbf{x}), \quad \text { where } 1 \leq i \leq n, 1 \leq j \leq m .
$$

Here, $x_{i j}$ is the concentration of the $i$ th species at the $j$ th level (e.g., a particular mRNA, protein or metabolite), $\mathbf{x}$ is the vector of concentrations of all the species at all levels, and $g_{i j}$ is a function, often involving thresholds and sigmoid curves. In dynamical control systems, output often becomes input, both within and across types of elements. It is important to distinguish between feedback and coupling. Goodwin (1963) provided one of the first models of gene control in what he called "the epigenetic system." He differentiated three levels of production in this system: (1) mRNAs, (2) proteins (usually enzymes), and (3) metabolites. Goodwin argued that gene regulation is a feedback loop: $(1) \rightarrow(2) \rightarrow(3) \rightarrow(1)$. A single idealized unit component $(1963,23)$ is one in which mRNA is linked only with its produced protein and metabolite. A general way of expressing the feedback loop of a unit component is

$$
\begin{gathered}
d x_{1} / d t=a_{1 n} r\left(x_{n}\right)-b_{1} x_{1}, \\
d x_{j} / d t=a_{j, j-1} x_{j-1}-b_{j} x_{j}, \quad \text { where } 1<j \leq m,
\end{gathered}
$$

where $a$ is a parameter of production, $b$ is a parameter of degradation, $r$ is a function mapping the concentrations of metabolites to the production of mRNA, and $x_{j}$ is the concentration of the particular species at the $j$ th level. Note that there is no indexed $i$ since it has a single value for a given unit component. The function $r$ is negative for the common case of repression. The concentration of species at each level (e.g., $j=2$ for proteins, $j=3$ for metabolites) depends on species concentrations at lower levels. 
Moreover, we need to consider more realistic situations where multiple unit components are connected, and the equations are coupled. For instance, this would occur when metabolite species $_{1}$ (e.g., repressor ${ }_{1}$ ) has some influence on $\mathrm{mRNA}_{2}$, or when metabolite species 2 partially controls $\mathrm{mRNA}_{1}$, or both. For these cases, the vector $\mathbf{x}$ of [7] has nonzero values in more than one column, where columns express species concentrations (i.e., there is now more than one $i$ ). The rate equations of each unit component are now coupled to other unit components and we would have to index $i$ in [8] and [9]. In short, coupled differential equations capture (i) feedback within unit components, and (ii) connections across unit components.

GRNs modeled through Boolean networks, coupled differential equations, or other methods, capture changes of each element of a system as a function of what other elements are doing, over time. A number of contemporary systems theorists take GRNs to be a convenient and powerful reducing theory ( $T_{1}$ in Schaffnerian terms) that helps explain and predict development and physiology. Here is Davidson: "Someday we are going to be able to write down the regulatory network architecture [GRN] for key features of development that are characteristic of animals representing diverse regions of the phylogenetic diagram. . . . Differences in the regulatory network architecture are what cause phylogenetic diversification, and therein resides the 'deep structure' that underlies ['phylogenetic'] diagrams" (2001, 23). Davidson is not the only one with high expectations (e.g., Hartwell et al. 1999; Regev and Shapiro 2002; Fell 2007). While we must remain critical of the development and application of mathematical GRN theory and modeling, it does hold significant promise.

What exactly do GRNs explain? There are at least two sorts of potential theory reduction of phenotypic models to GRN models $\left(T_{1}\right)$ : (1) qualitative (i.e., from $T_{2}$ ) and (2) mathematically abstracted (i.e., from $T_{2}^{*}$ ). First, some GRN models explain well-defined but qualitative phenotypic characters, such as the spatial and temporal order of developmental cellular and tissue differentiation in sea urchin embryos and Arabidopsis flowers. In this case, $T_{2}$ is a structured set of phenotypic characters. One example is the important $A B C$ model of qualitative phenotype of the flowers of Arabidopsis and other plant species (e.g., Coen and Meyerowitz 1991; Espinosa-Soto, Padilla-Longoria, and Álvarez-Buylla 2004). Second, other work on GRN abstracts explicit mathematical models of morphological and developmental phenotype (e.g., Salazar-Ciudad and Jernvall 2002; Prusinkiewicz et al. 2007). Such phenotypic models can be thought of as corrected models on analogy with Schaffnerian theory correction. Mathematically abstracted phenotypic models (i.e., $T_{2}^{*}$ ) are directly derived from, embedded in, and, ultimately, reduced to GRN models (i.e., $T_{1}$ ). 
Mathematical theory reduction may turn out to be only partial, yet it can produce explanations and predictions (Davidson 2001).

The suggested reconstructive project of Schaffner's model employs important philosophical tools surrounding the epistemology of mathematical practices: (1) the analysis of mathematical modeling by the semantic view of theories, and (2) the concept of mathematical embedding (e.g., Friedman 1981, 1983; van Fraassen 1989). Let us see how by returning to the second and third aspects of the project listed earlier in this section.

Derivational mathematical practice within and across theories can be legitimately interpreted as deductive. Thus, we can understand reduction functions in terms of mathematics rather than in terms of logic (e.g., Schaffner's extensional characterization of terms in different, logically formulated theories). Mathematical reduction functions can powerfully and justifiably: (1) map the same particular variables and parameters (i.e., term-like formalisms), and basic functions ("laws of the model") across mathematical theories and models of different mereological levels, or (2) directly correlate variables, parameters, or basic functions of $T_{1}$ models to different variables, parameters, or basic functions of $T_{2}$ or $T_{2}^{*}$ models. The state space approach of the semantic view provides useful resources for constructing a mathematical account of reduction functions that simultaneously focuses on the importance of modeling (van Fraassen 1970, 1972, 1989; Lloyd 1988; Weisberg 2009).

Moreover, embedding is a concept important to the generalization or mathematization, as it were, of deduction. In early work, Friedman argues that many scientific explanations "follow a common pattern": "observational structure[s]" are "construe[d] as substructures" of "theoretical structure[s]"; moreover, theoretical structures have "complex and interesting mathematical properties" (1983, 236; see also 1981). In discussing "embedding" as an "aspect" of theorizing, van Fraassen makes a similar point: "The phenomena are small but chaotic; they are treated as fragments of a 'whole' that is much larger but orderly and simple" (1989, 230; entire sentence emphasized in the original). Embedding is here considered a mathematical subsumption relation between theoretical and observational formal structures within a single theory or set of models. I suggest a hierarchical extension of the concept: embedding can also be effected across mathematical theories representing different levels of organization (n.b., each theory has theoretical and observational components, which are potentially overlapping and interacting). The mathematical practice of embedding can lead to increased unification of mathematical structure. Even though embedding has limits and biases (Winther 2006a; Wimsatt 2007), and although theoretical and observational structures of a mathematical theory cannot always be distinguished 
sharply, embedding across theories is an important reductionist research strategy and regulative ideal in science generally.

With a reconstructed model of mathematical theory reduction, light could also be shed on how to quantify conceptual distance. Metrics for measuring the formal similarity between mathematical theories representing different mereologically nested levels could be developed (e.g., "homeomorphism" in the sense of Lloyd 1988, or the extent of variable or law sharing). Theories with higher degrees of formal similarity are conceptually closer.

In conclusion, two caveats are important. First, it is perhaps unsurprising that something like a reconstructed Schaffner model has not been previously attempted. Recent analyses of the biological sciences as "mechanistic" (e.g., Glennan 1996, 2002; Machamer, Darden, and Craver 2000; Bechtel 2006; Craver 2007; see also Wimsatt 1976; Sarkar 1998; Schaffner 2006, 2007), "compositional" or "part-whole" (Winther 2006b, 2009), and "experimental" (Weber 2005) often diminish the relevance of mathematical practices for the life sciences. I do not here claim that mathematics will replace mechanisms, let alone compositionality or experiment. Rather, I see each of these as important, especially for the biological sciences. Second caveat: while mathematical derivation and embedding remain important regulative ideals, it is unclear whether we could ever expect an elegantly unified, global mathematical structure for the biological sciences. Indeed, cogent arguments have been given for the "dappled" structure of science (e.g., Cartwright 1983, 1999; Burian 1993; Schaffner 1993b; van der Steen 1993). But the jury is still out. I end with a question. Should the clear importance of mechanisms, part-whole relations, and experiment, as well as the possibility of irreducible dappledness, continue to stop us from even trying to understand the roles mathematical reduction, derivation, and embedding play in the biological sciences?

\section{REFERENCES}

Bagheri, H. C. (2005), "Unresolved Boundaries of Evolutionary Theory and the Question of How Inheritance Systems Evolve: 75 Years of Debate on the Evolution of Dominance", Journal of Experimental Zoology, Part B: Molecular and Developmental Evolution 306: 1-31.

Batterman, R. (2005), "Intertheory Relations in Physics", in Edward N. Zalta (ed.), The Stanford Encyclopedia of Philosophy, Winter 2005 ed., http://plato.stanford.edu/archives/ win2005/entries/physics-interrelate/.

Bechtel, W. (2006), Discovering Cell Mechanisms: The Creation of Modern Cell Biology. Cambridge: Cambridge University Press.

Beurton, P. J. (2000), "A Unified View of the Gene, or How to Overcome Reductionism", in P. J. Beurton, R. Falk, and H. J. Rheinberger (eds.), The Concept of the Gene in Development and Evolution: Historical and Epistemological Perspectives. Cambridge: Cambridge University Press, 286-314.

Brandon, R. (1997), "Does Biology Have Laws? The Experimental Evidence", Philosophy of Science 64 (Proceedings): S444-S457. 
Brigandt, I., and A. Love (2008), "Reductionism in Biology", in Edward N. Zalta (ed.), The Stanford Encyclopedia of Philosophy, Fall 2008 ed., http://plato.stanford.edu/archives/fall2008/entries/reduction-biology/.

Burian, R. M. (1993), "Unification and Coherence as Methodological Objectives in the Biological Sciences", Biology and Philosophy 8: 301-318.

Callender, C. (1999), "Reducing Thermodynamics to Statistical Mechanics: The Case of Entropy", Journal of Philosophy 96: 348-373.

Cartwright, N. (1983), How the Laws of Physics Lie. Oxford: Oxford University Press. (1999), The Dappled World: A Study of the Boundaries of Science. Cambridge: Cambridge University Press.

Coen, E. S., and E. M. Meyerowitz (1991), "The War of the Whorls: Genetic Interactions Controlling Flower Development", Nature 353: 31-37.

Cooper, G. (1996), "Theoretical Modeling and Biological Laws", Philosophy of Science 63 (Proceedings): S28-S35.

Craver, C. F. (2007), Explaining the Brain. Oxford: Oxford University Press.

Darden, L., and J. Tabery (2005), "Molecular Biology", in Edward N. Zalta (ed.), The Stanford Encyclopedia of Philosophy, Spring 2005 ed., http://plato.stanford.edu/archives/ spr2005/entries/molecular-biology/.

Davidson, E. (2001), Genomic Regulatory Systems: Development and Evolution. San Diego: Academic Press.

De Jong, H. (2002), "Modeling and Simulation of Genetic Regulatory Systems: A Literature Review", Journal of Computational Biology 9: 67-103.

Espinosa-Soto, C., P. Padilla-Longoria, and E. Álvarez-Buylla (2004), "A Gene Regulatory Network Model for Cell-Fate Determination during Arabidopsis thaliana Flower Development That Is Robust and Recovers Experimental Gene Expression Profiles", Plant Cell 16: 2923-2939.

Fell, D. (2007), "How Can We Understand Metabolism?", in F. C. Boogerd et al. (eds.), Systems Biology: Philosophical Foundations. Amsterdam: Elsevier, 87-101.

Feyerabend, P. (1962), "Explanation, Reduction, and Empiricism", in H. Feigl and G. Maxwell (eds.), Scientific Explanation, Space, and Time. Minnesota Studies in the Philosophy of Science, vol. 3. Minneapolis: University of Minnesota Press, 28-97.

_ (1965), "Problems of Empiricism", in R. G. Colodny (ed.), Beyond the Edge of Certainty: Essays in Contemporary Science and Philosophy. Englewood-Cliffs, NJ: Prentice-Hall, 145-260.

Field, H. (1973), "Theory Change and the Indeterminacy of Reference", Journal of Philosophy 70: 462-481.

Fodor J. (1974), "Special Sciences (or the Disunity of Science as a Working Hypothesis)", Synthese 28: 97-115.

Friedman, M. (1974), "Explanation and Scientific Understanding", Journal of Philosophy 71: 5-19.

- (1981), "Theoretical Explanation", in R. Healey (ed.), Reduction, Time, and Reality: Studies in the Philosophy of the Natural Sciences. Cambridge: Cambridge University Press, 1-16.

(1983), Foundations of Space-Time Theories: Relativistic Physics and Philosophy of Science. Princeton, NJ: Princeton University Press.

Gentner, D. (1983), "Structure-Mapping: A Theoretical Framework for Analogy", Cognitive Science 7: 155-170.

Glennan, S. (1996), "Mechanisms and the Nature of Causation", Erkenntnis 44: 49-71. (2002), "Rethinking Mechanistic Explanation", Philosophy of Science 69 (Proceedings): S342-S353.

Goodwin, B. (1963), Temporal Organization in Cells: A Dynamic Theory of Cellular Control Processes. New York: Academic Press.

Halonen, I., and J. Hintikka (2005), "Toward a Theory of the Process of Explanation", Synthese 143: 5-61.

Hartwell, L., J. Hopfield, S. Leibler, and A. Murray (1999), "From Molecular to Modular Cell Biology", Nature 402: C47-C52.

Hempel, C. (1966), Philosophy of Natural Science. Englewood Cliffs, NJ: Prentice-Hall. 
Hempel, C., and P. Oppenheim (1948), "Studies in the Logic of Explanation", Philosophy of Science 15: 135-175.

Holyoak, K. J., and P. Thagard (1989), "Analogical Mapping by Constraint Satisfaction”, Cognitive Science 13: 295-355.

Hull, D. (1972), "Reduction in Genetics-Biology or Philosophy?", Philosophy of Science 39: 491-499.

_ (1974), Philosophy of Biological Sciences. Englewood Cliffs, NJ: Prentice-Hall.

(1976), "Informal Aspects of Theory Reduction", in R. S. Cohen et al. (eds.), PSA 1974: Proceedings of the 1974 Biennial Meeting of the Philosophy of Science Association. Dordrecht: Reidel, 653-670.

- (1981), "Reduction and Genetics", Journal of Medicine and Philosophy 6: 125-143.

(2002), "Varieties of Reductionism: Derivation and Gene Selection", in M. Van Regenmortel and D. Hull (eds.), Promises and Limits of Reductionism in the Biomedical Sciences. London: Wiley, 161-177.

Kauffman, S. (1993), The Origins of Order: Self-Organization and Selection in Evolution. Oxford: Oxford University Press.

Kim, J. (1992), "Multiple Realization and the Metaphysics of Reduction", Philosophy and Phenomenological Research 52: 1-26.

Kitano, H. (2002), "Looking beyond the Details: A Rise in System-Oriented Approaches in Genetics and Molecular Biology", Current Genetics 41: 1-10.

Kitcher, P. (1981), "Explanatory Unification", Philosophy of Science 48: 251-281.

_ (1984), "1953 and All That: A Tale of Two Sciences", Philosophical Review 93: 335 373.

(1989), "Explanatory Unification and Causal Structure", Minnesota Studies in the Philosophy of Science 13: 410-505.

(1999), "Unification as a Regulative Ideal”, Perspectives on Science 7: 337-348.

Lange, M. (2000), Natural Laws in Scientific Practice. Oxford: Oxford University Press.

Lloyd, E. A. (1988), The Structure and Confirmation of Evolutionary Theory. Princeton, NJ: Princeton University Press.

Machamer, P., L. Darden, and C. F. Craver (2000), "Thinking about Mechanisms", Philosophy of Science 67: 1-25.

Markman, A. B. (1997), "Constraints on Analogical Inference", Cognitive Science 21: 373 418.

Mendoza, L., and E. Álvarez-Buylla (1998), "Dynamics of the Regulatory Genetic Network for Arabidopsis thaliana Flower Morphogenesis", Journal of Theoretical Biology 193: 307-319.

Mesarovic, M. D., ed. (1968), Systems Theory and Biology. New York: Springer-Verlag.

Nagel, E. (1949), "The Meaning of Reduction in the Natural Sciences", in R. Stauffer (ed.), Science and Civilization. Madison: University of Wisconsin Press, 99-145.

- (1961), The Structure of Science: Problems in the Logic of Scientific Explanation. London: Routledge.

(1979), "Issues in the Logic of Reductive Explanations", in Teleology Revisited and Other Essays in the Philosophy and History of Science. New York: Columbia University Press, 95-117.

Okasha, S. (2007), Evolution and the Levels of Selection. Oxford: Oxford University Press.

O’Malley, M. A., and J. Dupré (2005), "Fundamental Issues in Systems Biology", BioEssays 27: $1270-1276$.

Pemmaraju, S., and S. Skiena (2003), Computational Discrete Mathematics: Combinatorics and Graph Theory with Mathematica ${ }^{\circledast}$. Cambridge: Cambridge University Press.

Prusinkiewicz, P., Y. Erasmus, B. Lane, D. Harder, and E. Coen (2007), "Evolution and Development of Infloresence Architectures", Science 316: 1452-1456.

Psillos, S. (1999), Scientific Realism: How Science Tracks the Truth. London: Routledge.

Rashevsky, N. (1954), "Topology and Life", Bulletin of Mathematical Biophysics 16: 317 348.

(1961), Mathematical Principles in Biology and their Application. Springfield, IL: Thomas.

Regev, A., and E. Shapiro (2002), "Cells as Computation”, Nature 419: 343. 
Rosen, R. (1964), “Abstract Biological Systems as Sequential Machines”, Bulletin of Mathematical Biophysics 26: 103-111.

(1985), "Organisms as Causal Systems Which Are Not Mechanisms: An Essay into the Nature of Complexity", in Robert Rosen (ed.), Theoretical Biology and Complexity. San Diego: Academic Press, 165-203.

Rosenberg, A. (1978), "The Supervenience of Biological Concepts", Philosophy of Science 45: $368-386$.

(1985), The Structure of Biological Science. Cambridge: Cambridge University Press. (1997), "Reductionism Redux: Computing the Embryo", Biology and Philosophy 12: $445-470$

(2002), "Reductionism in a Historical Science", in M. Van Regenmortel and D. Hull (eds.), Promises and Limits of Reductionism in the Biomedical Sciences. London: Wiley, $125-160$.

(2006), Darwinian Reductionism; or, How to Stop Worrying and Love Molecular Biology. Chicago: University of Chicago Press.

Ruse, M. (1976), "Reduction in Genetics", in R. S. Cohen et al. (eds.), PSA 1974: Proceedings of the 1974 Biennial Meeting of the Philosophy of Science Association. Dordrecht: Reidel, 633-651.

Salazar-Ciudad, I., and J. Jernvall (2002), "A Gene Network Model Accounting for Development and Evolution of Mammalian Teeth", Proceedings of the National Academy of Sciences 99: 8116-8120.

Sarkar, S. (1992), "Models of Reduction and Categories of Reductionism", Synthese 91: $167-194$

- (1998), Genetics and Reductionism. Cambridge: Cambridge University Press.

(2001), "Reductionism in Genetics and the Human Genome Project", in R. S. Singh et al. (eds.), Thinking about Evolution: Historical, Philosophical, and Political Perspectives, vol. 2. Cambridge: Cambridge University Press, 235-252.

Schaffner, K. F., (1967), "Approaches to Reduction", in Philosophy of Science 34: 137-147. (1969), "The Watson-Crick Model and Reductionism", British Journal for the Philosophy of Science 20: 325-348.

(1976), "Reductionism in Biology: Prospects and Problems", in R. S. Cohen et al. (eds.), PSA 1974: Proceedings of the 1974 Biennial Meeting of the Philosophy of Science Association. Dordrecht: Reidel, 613-632.

(1993a), Discovery and Explanation in Biology and Medicine. Chicago: University of Chicago Press.

_ (1993b), "Theory Structure, Reduction, and Disciplinary Integration in Biology", Biology and Philosophy 8: 319-347.

(2006), "Reduction: The Cheshire Cat Problem and a Return to Roots", Synthese 151: $377-402$.

(2007), "Theories, Models, and Equations in Systems Biology", in F. C. Boogerd (ed.), Systems Biology: Philosophical Foundations. Amsterdam: Elsevier, 145-162.

Shimony, A. (1987), "The Methodology of Synthesis: Parts and Wholes in Low-Energy Physics", in R. Kargon and P. Achinstein (eds.), Kelvin's Baltimore Lectures and Modern Theoretical Physics. Cambridge, MA: MIT Press, 399-423.

Sklar, L. (1999), "The Reduction (?) of Thermodynamics to Statistical Mechanics", Philosophical Studies 95: 187-202.

Smolen, P., D. A. Baxter, and J. H. Byrne (2000), "Modeling Transcriptional Control in Gene Networks-Methods, Recent Results, and Future Directions", Bulletin of Mathematical Biology 62: 247-292.

Sober, E. (1997), "Two Outbreaks of Lawlessness in Recent Philosophy of Biology", Philosophy of Science 64 (Proceedings): S458-S467.

_ (1999), "The Multiple Realizability Argument against Reductionism", Philosophy of Science 66: 542-564.

Thomas, R. (1973), "Boolean Formalization of Genetic Control Circuits", Journal of Theoretical Biology 42: 563-585.

Tyson, J. J., and H. G. Othmer (1978), "The Dynamics of Feedback Control Circuits in 
Biochemical Pathways", in R. Rosen and F. M. Snell (eds.), Progress in Theoretical Biology, vol. 5. New York: Academic Press, 1-62.

van der Steen, Wim J. (1993), “Towards Disciplinary Disintegration in Biology”, Biology and Philosophy 8: 259-275.

van Fraassen, B. (1970), "On the Extension of Beth's Semantics of Physical Theories", Philosophy of Science 37: 325-339.

- (1972), "A Formal Approach to the Philosophy of Science", in R. Colodny (ed.), Paradigms and Paradoxes: The Philosophical Challenge of the Quantum Domain. Pittsburgh: University of Pittsburgh Press, 303-366.

(1989), Laws and Symmetry. Oxford: Oxford University Press.

von Bertalanffy, L. ([1933] 1962), Modern Theories of Development: An Introduction to Theoretical Biology. New York: Harper Torchbooks.

(1968), General System Theory. Foundations, Development, Applications. New York: Braziller.

Waters, C. K. (1990), "Why the Antireductionist Consensus Won't Survive the Case of Classical Mendelian Genetics", in A. Fine, M. Forbes, and L. Wessels (eds.), PSA 1990: Proceedings of the 1990 Biennial Meeting of the Philosophy of Science Association, vol. 1. East Lansing, MI: Philosophy of Science Association, 125-139.

(1998), "Causal Regularities in the Biological World of Contingent Distributions", Biology and Philosophy 13: 5-36.

(2000), "Molecules Made Biological", Revue Internationale de Philosophie 4 (214): 539-564.

Weber, M. (2005), Philosophy of Experimental Biology. Cambridge: Cambridge University Press.

Weisberg, M. (2009), "Models for Modeling", in preparation.

Wimsatt, W. C. (1976), "Reductive Explanation: A Functional Account", in R. S. Cohen et al. (eds.), PSA 1974: Proceedings of the 1974 Biennial Meeting of the Philosophy of Science Association. Dordrecht: Reidel, 671-710.

(2007), Re-engineering Philosophy for Limited Beings: Piecewise Approximations to Reality. Cambridge, MA: Harvard University Press.

Winther, R. G. (2006a), "Fisherian and Wrightian Perspectives in Evolutionary Genetics and Model-Mediated Imposition of Theoretical Assumptions", Journal of Theoretical Biology 240: 218-232.

(2006b), "Parts and Theories in Compositional Biology", Biology and Philosophy 21: $471-499$

(2008), "Systemic Darwinism", Proceedings of the National Academy of Sciences 105: 11833-11838.

(2009), "Part-Whole Science", Synthese, forthcoming. 